\title{
What is Creative Leadership?
}

By Dr Ninela Ivanova

The term "creative leadership" has significantly gained traction over the last decade. It is often associated with a style of leadership that encourages creativity in devising new business strategies and models, in organizational development and change management, in building environments and teams that use creative processes in innovating products and services. In the creative industries, creative leadership is synonymous with visionary thinking and being a pioneer in a design or a creative field. It hopes to bring to the fore a new set of values and practices and reconnect with what it truly means to be human, to create, co-create, and collaborate with others, and to develop new capacities that enable us to take the lead in designing new ways of living, working, and connecting. "What futures, what worlds do we want to create?" would be a better question.

In all cases, engagement with an artistically creative mindset is of the essence, and in 2010, an IBM study of global CEOs ${ }^{1}$ established that business leaders themselves acknowledged creativity as the foremost leadership quality that required attention and development. In recent years, this has formed the basis for new leadership and innovation programs that teach creative thinking and skills, with a view to "unlocking leadership potential."

My role in Creative Leadership at the Helen Hamlyn Centre for Design has been to build on the existing body of work in the areas of empathy, clarity, and creativity, and to establish a rich evidence-base for future creative leadership theory-building, research and applications. Over the last 16 months, this has entailed various activities spanning qualitative and quantitative research, development of design briefs, delivery of workshops, and executive education. Key questions that guided this process included:

- What are the components of a creativity mindset?

- How do you teach these capacities so that they become entrained in the individual, rather than a prescribed solution or strategy?

- How do you apply different creative processes on a moment-by-moment basis - contextually, with intelligence and sensibility, and for the betterment of all?

- How can we measure the values — empathy, clarity, and creativity —of Creative Leadership? What are their essential cognitive, emotional and behavioral components?

- What types of education and training would elicit activation of specific patterns of cognition and behavior on an individual and collective level?

- What design methods and tools can be applied to bring about the activation of these processes?

- What factors enable or preclude the development of Creative Leadership on a personal, collective and global scale?

${ }^{1}$ IBM Corp., "The Enterprise of the Future," 2010. 
In addressing the above questions, we developed a program of research and delivery that would enable individuals and groups to develop the specific cognitive, emotional, and behavioral capacities that are needed to thrive and succeed in a world of relentless volatility, uncertainty, complexity, and ambiguity. Together with neuroscientist Dr Melanie Flory, who joined the Creative Leadership team in April 2018, we designed and ran a pilot study to test how people experienced the concepts of empathy, clarity, and creativity in leadership and their day-to-day work.

An ongoing aspect of the research is based on a series of interviews with leaders and senior management across the public, private, and policy sectors to examine whether components of creative leadership are already embedded (and recognized) within current leadership styles and practices. Below are some "takeaways" from this research.

\section{There is a collective call for "mavericks" in leadership roles.}

Although tradition, hierarchy, structure, centralized power, and being target-focused are seen as important in ensuring a cohesive, value-based culture and accountability in execution, there is a need for new modes of leadership that disrupt old patterns and business models, linear company structures, siloes and roles, global markets and narratives; and enable more collaborative, inclusive, and multi-disciplinary ways of living and working.

\section{A new organizational culture is emerging.}

Leaders I interviewed acknowledge that bringing out the leadership in others, "letting their team shine through," is important to their leadership style and celebrated by their organizations. A coaching mindset and principles are becoming embedded into organizations, to serve internal teams and clients alike. Incorporating play and experimentation in day-to-day work practices is seen as a way to enable a more inventive and co-operative corporate culture.

\section{Being empathic doesn't mean soft.}

Empathy, as used in the design field, has specific associations with being people-centered, engaging with end clients, and actively listening for the real need or aspiration in the market. It is in the blueprint of great design.

In business, being empathic is not always seen as a plus. You may be perceived as a "pushover," "too emotional," "not professional." This is a misunderstanding of empathy, and its power. When we look at specific aspects of empathy, far from it being a personality trait, it becomes an intelligence and a practice that can be taught. For example, male leaders report that they recognize the need for diversity in senior management and at board level but struggle to implement, or even imagine, a long-term plan of action. Training in listening, humility, and kindness could spark off new conversations towards a more inclusive and sustainable leadership landscape.

\section{Clarity does not preclude creativity.}

We are moving away from design-indulgent practices, i.e., design for design's sake, or creativity for creativity's sake. Understanding how a new product or service addresses a real need in the market has always been at the heart of inclusive design, and is also a value in creative leadership. Calling for clarity 
of purpose, communication, project scope, roles and deliverables, and aligning intent, processes, methods and teams, is essential to success.

\section{Good leaders are good communicators.}

Enabling and establishing "shared meaning" is a primary objective of communication, especially in a world where digital technologies are bringing together multi-lingual, multi-cultural, multi-disciplinary organizations and teams. We can no longer afford to assume that we share the same understanding of vision, terms, and objectives. Transparency, openness, and listening are becoming key values.

\section{Some final thoughts}

Empathy, clarity, and creativity are not constant values. They can vary on a day-to-day basis depending on situation, context, and environment. Our research to date shows a real need for refining and expanding definition of these terms, and enabling understanding of their multiple applications in enterprise and education. They are not abstract concepts, or principles, but a spectra of capacities and attributes that can be exercised and developed.

This is where the pairing of design and neuroscience can add real value. While designers are uncovering latent needs and pathways to application, scientists can contribute an advanced understanding of empathy, clarity, and creativity that can be trained in individuals and groups. For instance, neuroscientist Flory asserts that exercising these skills actually drives functional changes in the brain.

Lastly, barriers can be overcome through continued practice. There are multiple barriers to creative leadership - from egocentric mindset and stubbornness to a lack of understanding of self-worth and value; from emotional barriers (fear and stress) to external factors (time, resources, and technology). When barriers arise, people easily revert to their default modes of practice and behaviors. We call for continued practice and contextual application, so that empathy, clarity, and creativity can become part of the make-up of the individual, and "intelligences" in their own right. 